\title{
Superoleophobic and Conductive Carbon Nanofiber/Fluoropolymer Composite Films
}

\author{
Arindam Das ${ }^{a}$, Thomas M. Schutzius ${ }^{a}$, Ilker S. Bayer ${ }^{b, c}$, Constantine M. Megaridis, ${ }^{a, *}$ \\ ${ }^{a}$ Department of Mechanical and Industrial Engineering, University of Illinois at Chicago, Chicago, IL 60607, USA \\ ${ }^{b}$ Center for Biomolecular Nanotechnologies, Smart Materials Platform, Italian Institute of \\ Technology@UNILE,Arnesano,LE 73010, Italy \\ ${ }^{c}$ Department of Mechanical and Aerospace Engineering, University of Virginia, Charlottesville, VA 22904, USA
}

\begin{abstract}
A solution-based, large-area coating procedure is developed to produce conductive polymer composite films consisting of hollow-core carbon nanofibers (CNFs) and a fluoroacrylic copolymer available as a water-based dispersion. CNFs (100nm dia., length 130 $\mu$ m) were dispersed by sonication in a formic acid/acetone co-solvent system, which enabled colloidal stability and direct blending of the CNFs and aqueous fluoroacrylic dispersions in the absence of surfactants. The dispersions were sprayed on smooth and microtextured surfaces, thus forming conformal coatings after drying. Nanostructured composite films of different degrees of oil and water repellency were fabricated by varying the concentration of CNFs. The effect of substrate texture and CNF content on oil/water repellency was studied. Water and oil static contact angles (CA) ranged from $98^{\circ}$ to $164^{\circ}$ and from $61^{\circ}$ to $164^{\circ}$, respectively. Some coatings with the highest water/oil CAs displayed self-cleaning behavior (droplet roll-off angles $<10^{\circ}$ ). Inherent conductivity of the composite films ranged from 63 to $940 \mathrm{~S} / \mathrm{m}$ at CNF concentrations from 10 to 60 wt. \%, respectively. Replacement of the long CNFs with shorter solid-core carbon nanowhiskers (150 nm dia., length 6-8 $\mu \mathrm{m}$ ) produced stable fluoropolymer-nanowhisker
\end{abstract}


dispersions, which were ink-jetted to generate hydrophobic, conductive, printed line patterns with a feature size $\sim 100 \mu$ m.

* Corresponding author. Tel: +1 312996 3436. E-mail address: cmm@uic.edu (C. M. Megaridis)

\section{Introduction}

Stable polymer-particle dispersions suitable for spray casting of nanostructured carbon composite coatings are important for a variety of industries seeking simple methods to apply large-area, functional surface treatments [1-9]. Such dispersions, when deposited on a suitable substrate and dried, can produce composite films with tunable mechanical, electrical, and hydrophobic/hydrophilic properties [4, 5]. Select methods for producing dispersions have focused on blending solution-processed polymers or aqueous based polymeric dispersions with dilute nanofiber suspensions [10]. Polymers are generally chosen based on their adhesion properties, mechanical strength, and surface energy, while nanoparticle fillers are selected not only to alter surface energy and surface texture, but also to impart additional functionalities, such as electrical conductivity, magnetic actuation, etc. Nanofillers, such as carbon nanotubes (CNTs) or carbon nanofibers (CNFs), in order to be combined with polymer dispersions or solutions must be dispersible and stable in liquids, which generally requires the use of either sonication, chemical functionalization, co-solvents or a combination of these techniques [11]. CNFs have the advantage of being less costly for industrial scale production [12]. But CNFs are also very long ( $>100 \mu \mathrm{m})$ - as compared to CNTs $(<10 \mu \mathrm{m})$ - which complicates their solution dispersion and processing. Relatively few works have demonstrated water repellency of composite coatings 
containing CNFs or CNTs dispersed in fluorinated polymers [5, 13]. Moreover, studies demonstrating CNF-based polymer composites with oil repellent properties are lacking.

Herein we report fabrication of novel CNF-fluoropolymer composite films obtained from multi-solvent dispersions comprising formic acid, acetone and water. Prior to blending CNFs into the aqueous-based fluoropolymer dispersion, CNFs are dispersed in a formic acid/acetone solvent system by ultrasonic processing. The fluoropolymer used is a commercially available fluoroacrylic co-polymer (PMC), which provides better substrate adhesion when compared to traditional perfluorinated polymers, such as polytetrafluoroethylene (PTFE or Teflon). The highaspect-ratio CNFs used in this work serve two purposes, namely to: a) create hierarchical surface roughness features within the polymer matrix as required for liquid repellency [14], and b) add additional functionality of electrical conductivity by a percolated fiber network when present in high enough concentrations. To enable solution dispersion, instead of treating CNFs with strong acids, such as sulfuric or trifluoroacetic acid [11], we use formic acid, a relatively weak carboxylic acid, which is completely compatible with the water-PMC dispersion, and hence facilitates efficient mixing of CNFs in polymer dispersions. Using this approach we obtain a number of such dispersions capable of producing superhydrophobic (water sessile contact angle, CA $>150^{\circ}$ ), self-cleaning (sliding angle $<10^{\circ}$ ), and superoleophobic (oil sessile CA $>150^{\circ}$ ) composite coatings when spray cast and dried on either smooth or low-cost textured surfaces (i.e., sand paper). Using sand paper of varying grit sizes, conformal coatings with different concentrations of CNFs were fabricated featuring different micro and nanoscale roughness. Nanoscale features were tuned by using different CNF concentrations in the polymer matrix.

In the following, we study the influence of micro/nanoscale roughness of these coatings on sessile contact angle and roll-off angle for both water and oil. Finally, we demonstrate, as an 
example, the application of the present technique with an alternative carbon nanofiller; by replacing the long hollow-core CNFs with similar diameter but shorter solid-core carbon fibers (henceforth termed nanowhiskers), PMC-carbon nanowhisker inks were prepared and ink-jetted producing linear patterns with feature size $\sim 100 \mu \mathrm{m}$. These inks can be formulated to display good substrate adhesion and electrical conductivity, in addition to their water and oil repellent properties, which are envisioned for the microelectronics industry. To date, only CNT-based suspensions (no polymer matrix) have been demonstrated for ink-jet applications (e.g. thin film transistors, etc.) with no studies reported on the wettability or adhesion of the deposited ink patterns [15-25].

\section{Experimental}

\subsection{Materials and Preparation of CNF/PMC composite}

Carbon nanofibers (PR-24-XT-HHT; Pyrograf III) were obtained from Applied Sciences Inc., USA. These CNFs were hollow with partially cup-and-cone structured graphitic walls and well characterized pretreatment-dependent mechanical properties [26]. Typical diameter of these asreceived CNFs was $100 \mathrm{~nm}$ with characteristic length $~ 130 \mu \mathrm{m}$; see Supplementary Information. The PMC was obtained from DuPont as a 20 wt. \% dispersion in water (Capstone ${ }^{\circledR}$ ST-100). Reagent grade formic acid, acetic acid, and acetone were obtained from Sigma Aldrich (USA) and used as received. Deionized water and mineral oil (White, Heavy; Sigma Aldrich Product \#330760; surface tension $28.54 \mathrm{mN} / \mathrm{m}$, density $0.862 \mathrm{~g} / \mathrm{cc}$ ) were used as probe liquids for contact and roll-off angle measurements.

The CNF dispersions were prepared as follows. Initially CNFs were dispersed in formic acid in five different concentrations of $0.5,1.0,1.5,2$ and 3 wt. \%, using a high power probe 
ultrasonic processor (Vibra-Cell, VCX-750, Sonics \& Materials, Inc.) operated at $70 \%$ amplitude for 5 minutes. Subsequently, an equal volume of acetone was added to the CNF dispersions and sonication was continued for another 5 minutes. It was found that sonication not only separated the CNF clusters, but also broke down the initially long fibers to lengths within the range 10-30 $\mathrm{mm}$. Separately, as-received aqueous PMC dispersion was diluted by mixing with formic acid and acetone to obtain a volume ratio of 1:1:2 (20 wt. \% PMC in water : formic acid : acetone). Diluted PMC and each of the five different CNF-in-formic-acid dispersions were then mixed in such proportion that the CNF content in the final dispersion was either 10, 20, 30, 40 or 60 wt. \% of the PMC polymer. The final CNF/PMC dispersions were applied by spray deposition on microscope glass slides and on sand paper of varying grit sizes corresponding to different microscale roughness. An airbrush atomizer (Paasche, VL siphon feed, $0.55 \mathrm{~mm}$ spray nozzle) was used to spray the dispersions. Spray cast films were dried at $90^{\circ} \mathrm{C}$ under a convective air flow space heater for 30 minutes. Care was exercised to control the final thickness of the composite films on sand paper so that they conform to the micro-texture of the underlying terrain rather than completely smoothen the substrate. Specifically, four spray passes were used to apply thin conformal coatings on sand paper. In this case, an upper bound estimate of the average coating thickness was $5 \mu \mathrm{m}$, as based on the projected area of the substrate (an underestimation of the actual textured surface area) and the mass of deposited dry CNF/PMC material. Thicker films, on the other hand, required several tens of spray passes over the smooth glass slides used for the electrical conductivity measurements.

Stable PMC-carbon nanowhisker dispersions for ink-jetting were produced by a similar process. Carbon nanowhiskers (150 nm dia., 6-8 $\mu \mathrm{m}$ long; see Supplementary Information) were procured from Sigma-Aldrich, USA. The nanowhiskers were first combined with acetone and 
sonicated for 10 min. Next, acetic acid (an alternative carboxylic acid) was introduced into the suspension and the sonication was continued for another 10 min. Finally, 20 wt. \% PMC in water was added to the suspension and sonication was maintained for $10 \mathrm{~min}$. For ink-jetting, the final dispersion was diluted with water to a level such that the collective concentration of carbon nanowhiskers and PMC in the solution was less than or equal to 1.5 wt. \%. Ink jetting was performed by a MicroFab JetLab ${ }^{\circledR} 4$ XL system equipped with a $60 \mu \mathrm{m}$ diameter nozzle.

\subsection{Coating Characterization}

Composite film thickness was measured using a calibrated high-quality optical microscope (OLYMPUS-BX51). Conductivity of CNF/PMC composite films deposited on glass slides was measured by a Keithley 6517 electrometer using the two-probe technique. Silver paint electrodes were applied locally on the films to minimize contact resistance between the films and the probes. A JEOL JEM-3010 (300 kV) instrument was used to obtain high resolution transmission electron microscope (TEM) images of the particle fillers. A scanning electron microscope (Philips XL30 ESEM-FEG) was used to analyze the surface morphology of the dry films. A backlit optical image setup employing a high speed camera (Red Lake Motion Pro, USA) and a tilt stage were used to measure sessile droplet CAs and liquid mobility on the composite films. A micro-syringe was used to dispense 6-8 $\mu$ l droplets of deionized water or mineral oil on the treated surfaces. Measurements were carried out by placing sessile water or oil droplets on a horizontal surface and subsequently tilting it until roll-off occurred. The droplet roll-off angle was defined as the minimum tilt angle required for droplet rolling. In many cases, especially with oil, roll-off did not occur even at $90^{\circ}$ tilt. At least ten different CAs and roll-off angle measurements were made at different locations on each of the CNF/PMC composite coatings. 
The present approach relies on static CA to designate super-repellency and on droplet roll-off angle to assess liquid mobility (the ability of a surface to shed liquid). The static value of CA is smaller than its advancing counterpart, which is suggested in [27] as a more reliable measure of repellency. To this end, the value of static CA offers a more conservative criterion for super-repellency (i.e. CA $>150^{\circ}$ ). As also reported in [27], liquid mobility correlated with the difference between the forward (maximum) and rear (minimum) CAs in a sliding droplet configuration on an inclined plate. However, this approach is not possible in the present study, as in many cases the droplets (especially oil) did not slide down the tilted surfaces, but rather remained stuck to them. We thus resorted to measure droplet roll-off angle, which offers an unambiguous criterion of liquid mobility on a surface when droplet rolling occurs.

\section{Results and Discussion}

\subsection{Dispersion Considerations}

Superoleophobic composite design and fabrication is considerably more difficult to achieve than superhydrophobicity, mainly because the former requires the creation of special hierarchical surface texture using very low surface energy materials [28]. For instance, micro-roughness having high degree of tip curvature, such as micro hoodoos or mushroom like features, is required instead of simple micron sized beads (bumps) so that low surface energy liquids (e.g. oils) can be maintained at a metastable Cassie-Baxter (non-wetting) state rather than the Wenzel (wetting) state. High-temperature-treated CNFs having highly graphitic walls are inherently hydrophobic [29]. Practically, high aspect ratio nanostructured materials can be self-assembled or grown into hierarchical structures (in the absence of a binding polymer matrix), which can display self-cleaning behavior, also known as the lotus effect [30]. In general, however, due to 
their high surface energy, such nanostructured materials (such as exfoliated clay platelets) need to be surface functionalized with hydrophobic macromolecules to display the lotus effect. Nanostructured carbon materials, such as CNFs and CNTs, can assemble into such structures provided that they are carefully grown in a unidirectional manner on select surfaces (i.e., CNT nanoforests on silicon wafers) from precursors using vapor deposition [31]. But these structures are delicate and lack durability due to the absence of an embedding and adherent polymer matrix. Simple and cost-effective techniques to fabricate durable and reusable CNF- or CNT-in-polymer composite films displaying the lotus effect are being sought because these nanomaterials are also electrically conductive [32]. There are two main problems to overcome: a) Difficulties in creating efficient and stable dispersions of long CNFs in a suitable solvent/polymer system, and b) identifying the right combination of surface morphology and surface energy of polymer matrix and filler material to cause lotus behavior. Owing to the hydrophobic nature and high aspect ratio of high-temperature-treated CNFs, their direct dispersion in water alone is very challenging. Addition of polar and water miscible solvents, such as acetone, may improve dispersion, but even in that case, CNFs begin to agglomerate and precipitate in solution at a fast rate. It has been reported [11] that introduction of TFA (trifluoroacetic acid) as a co-solvent improves the stability and dispersibility of CNTs in various organic solvents. In the present case, we demonstrate good performance with a much less aggressive carboxylic acid, namely formic acid, as co-solvent to disperse long CNFs efficiently. CNFs can be dispersed in formic acid to a certain extent. They still form large agglomerates in time, which practically hinders casting of films of uniform morphology. To this end, it is important to choose a polymer that will not only provide liquid repellency for the resulting composite coating, but also facilitate a high degree of CNF dispersibility and stability in the continuous phase prior to spraying. The PMC polymer 
used in the present work has amine-based acrylic cationic (hydrophilic) and fluorinated (hydrophobic) segments [33]. The acrylic segment promotes better adhesion, as compared with its liquid-repellent, perfluorinated counterpart. In the presence of water, the hydrophobic side chains of the polymer tend to orient towards the hydrophobic wall of the CNFs (Fig.1). The pH value of the dispersion has a critical role in charging of cationic segments associated with the PMC chemistry, and hence on the stabilization of the dispersion. In the absence of formic acid, the $\mathrm{pH}$ is very close to neutral as only water and acetone co-solvents are present in the dispersions. Under such nearly neutral $\mathrm{pH}$ conditions, the polymer is not properly ionized, and as a result, the CNFs tend to come close to each other and eventually form large aggregates in the co-solvent, as schematically shown in Fig. 1 (top row).
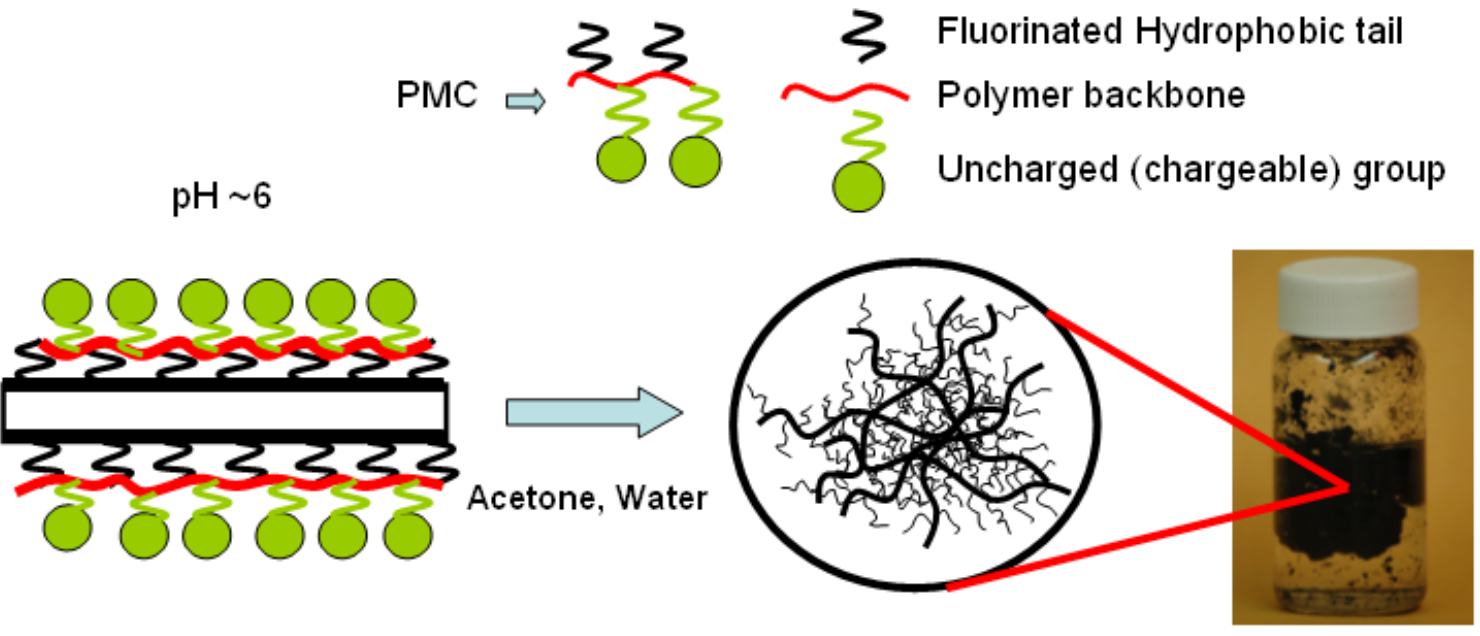

$$
\mathrm{pH} \sim 2
$$
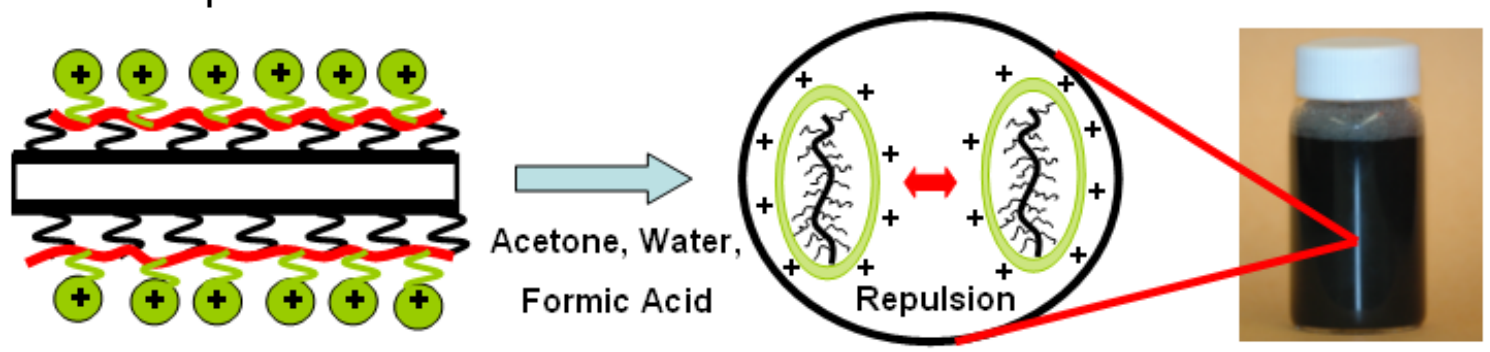
Fig. 1 - Schematic of the effect of formic acid on CNF dispersion stability. CNFs phase separate in the absence of formic acid (top panel), whereas CNFs disperse and produce ink like stable dispersions (bottom panel) in the presence of formic acid.

Introduction of formic acid into the system reduces the $\mathrm{pH}$ of the multicomponent solvent and starts ionizing the PMC. This allows CNFs with adsorbed PMC to be stabilized and separated by charge repulsion forces, which oppose hydrophobic interactions. Thus, aggregated CNFs start to disperse in the suspension after formic acid addition (Fig. 1 bottom row). Ultrasonic processing drastically improves this dispersion further by breaking up the aggregated CNFs.

To further assess the role of PMC on CNF dispersion quality in the acetone/water/formic acid solvent system (continuous phase), dispersions were prepared with formic acid as described above, but without PMC. The left vial in Fig. 2 contains a dispersion with 1 wt. \% CNF in an equal volume mixture of acetone, water and formic acid. When the vial was shaken mechanically after the dispersion was prepared, small CNF agglomerate deposits started to appear on the vial walls (red circled region in Fig. 2). Although the size and occurrence frequency of these agglomerates is much smaller than that in water or water/acetone mixtures in the absence of PMC, these agglomerates drastically reduce sprayability. On the other hand, when the CNF dispersions were prepared with PMC, no aggregate deposits on the vial walls were noticed (see right vial in Fig. 2) and dispersion sprayability was very efficient and repeatable. It is thus concluded that the role of PMC in obtaining an aggregate-free suspension is critical. 


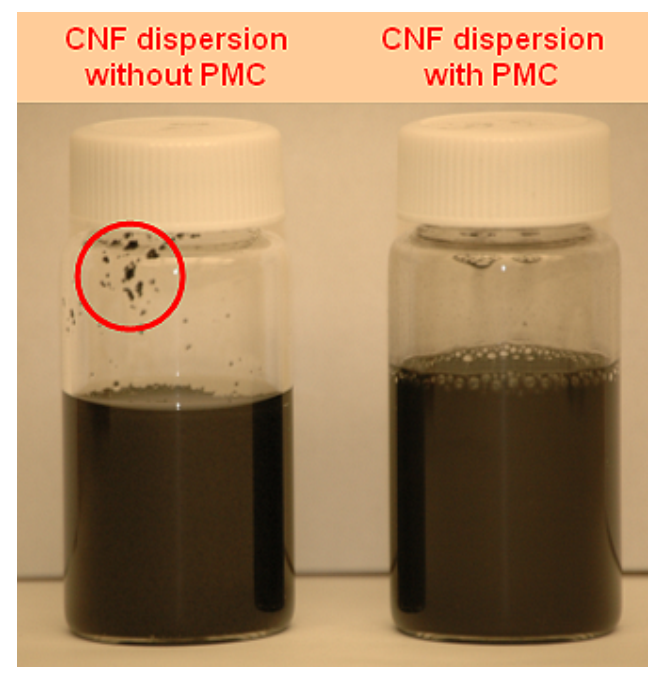

Fig. 2 - Role of PMC during CNF dispersion in acetone, formic acid and water mixture. (Left) Without PMC, large aggregates form (red circle); (Right) With PMC, superior CNF separation prevents aggregate formation.

Analysis of TEM images (Fig. 3) of CNFs extracted from PMC-free (control) and PMCcontaining dispersions indicate that PMC forms a thin polymeric layer on the CNF walls (Fig. 3b), which can further facilitate CNF dispersion. In the presence of formic acid in solution, adsorbed PMC could be charged since the $\mathrm{pH}$ of the continuous phase is lowered by the acid. In other words, the presence of formic acid increases the cationic behavior $(\mathrm{pH} \sim 2.3)$ of the adsorbed polymer layer, which in turn, makes the CNF walls positively charged. Hence, CNFs start to repel each other and thus overcome the forces associated with hydrophobic interactions, eventually dispersing efficiently in the continuous phase [34]. It is important to note that formic acid did not chemically functionalize or oxidize the CNF walls, as indicated by the absence of distortions on the walls of formic acid-treated CNFs (Fig. 3a). Such distortions have been previously observed after exposure of CNFs to nitric acid. 

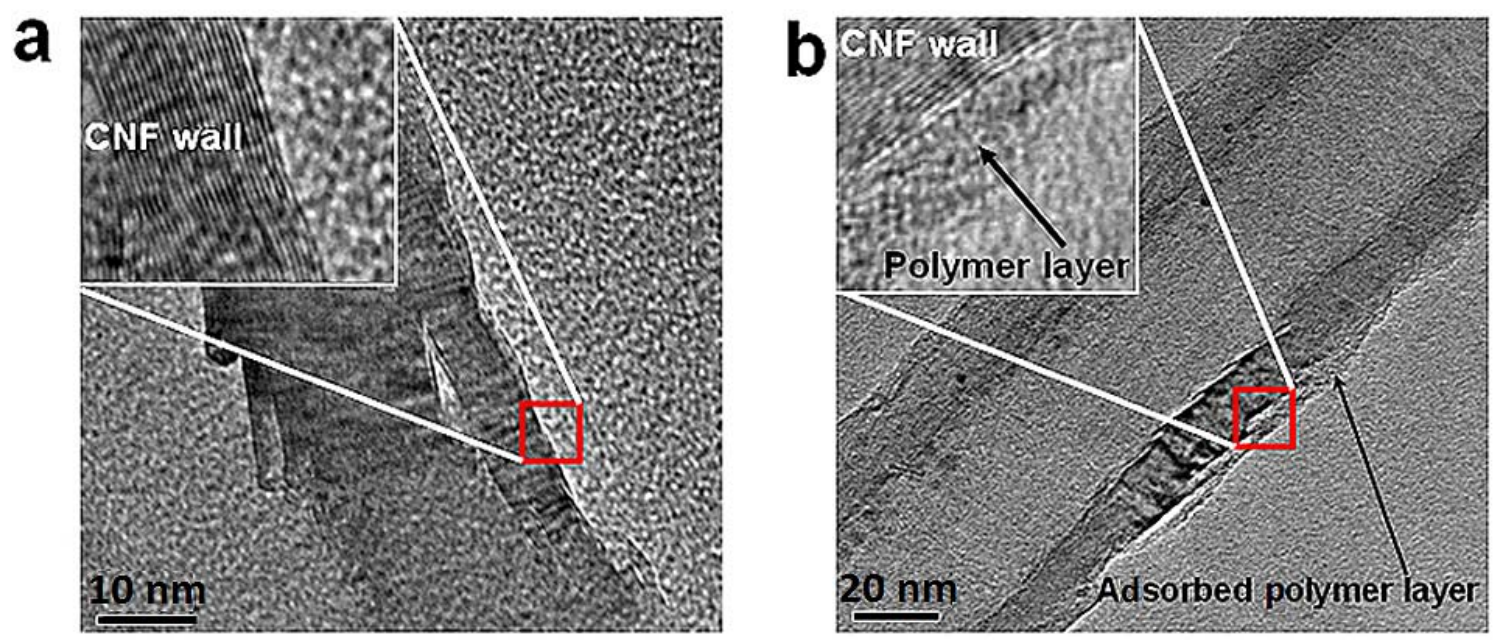

Fig. 3 - High resolution TEM image of CNF walls (a) after formic acid treatment; inset shows enlargement of intact graphene layers of CNF wall, indicating no chemical modification; (b) after treatment with formic acid and fluoropolymer dispersion diluted to $0.01 \mathrm{wt} \%$; inset shows detail of polymeric layer adsorbed over the outer CNF wall composed of intact graphene layers.

\subsection{Coating Surface Morphology}

The surface micro morphology of a thick $(150 \mu \mathrm{m})$ composite film deposited on smooth paper and containing 25 wt. \% CNFs in the PMC polymer matrix is shown in the SEM micrographs of Fig. 4. The film surface appears to be made up of randomly distributed micro bumps of different sizes and shapes (Fig. 4a) resembling the lotus leaf surface morphology. Surprisingly, the higher magnification image (Fig. 4b) indicates that these bumps are in fact made up of CNF bundles bound together by the polymer matrix. Therefore, CNFs form the required re-entrant, sub-micron roughness features within the larger (micron-scale) texture in these composites, thus causing high static water/oil contact angles. 

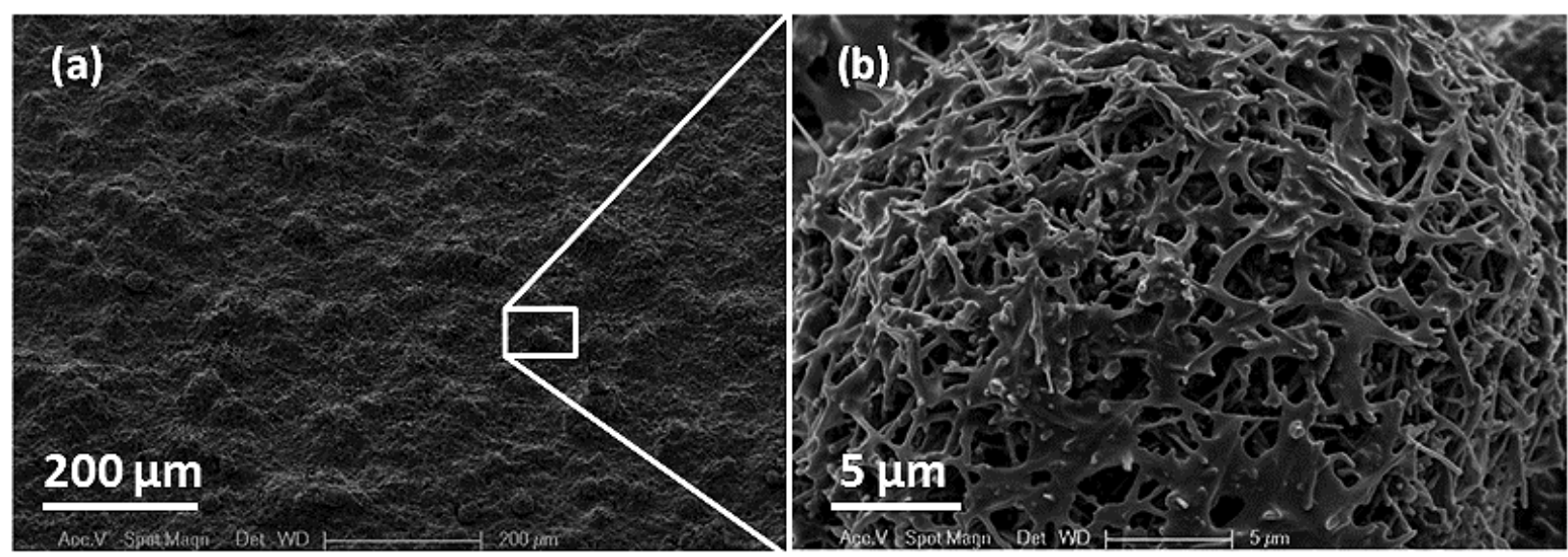

Fig. 4 - Scanning electron microscope images showing surface morphology of a thick coating (on smooth paper) comprising 25 wt. \% CNFs within the PMC polymer matrix. (a) Low magnification surface topology, and (b) enlargement of sub-micron surface roughness due to CNF bundles held together by the polymer.

(a)

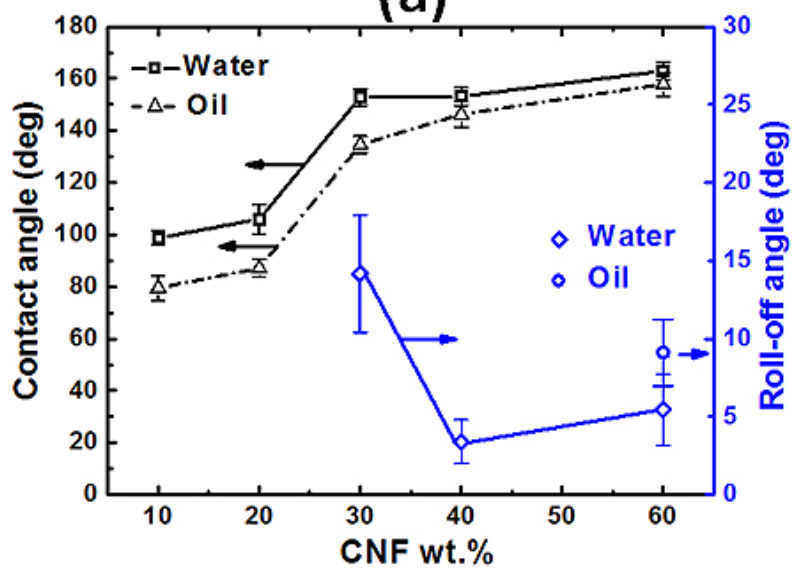

(b)

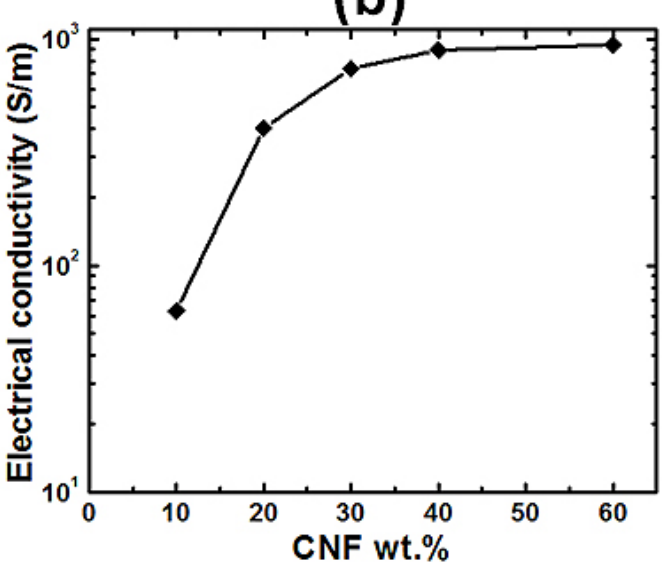

Fig. 5 - Variation of (a) sessile contact angle and droplet roll-off angle for water and oil, and (b) electrical conductivity with CNF loading for thick composite coatings applied on smooth glass slides.

\subsection{Large-Area Coating Wettability and Effect of Underlying Substrate Roughness}

The liquid repellent properties of spray cast CNF/PMC composite coatings applied on glass

slides and having varying CNF concentrations are reported in Fig. 5a, where static water and oil contact angles are plotted along with water and oil droplet roll-off angles as a function of CNF concentration (in wt. \% of the dried composite coating). For both water and oil, sessile contact angle increases and levels off with increasing CNF content. A transition from hydrophobic to 
superhydrophobic state is observed when CNF concentration reaches $30 \mathrm{wt}$ \%. The same trend is apparent for oil, where superoleophobicity (CA $>150^{\circ}$ ) is reached at 60 wt.\% CNF concentration. Water droplet mobility (rolling) is observed at a minimum CNF concentration of $30 \mathrm{wt} . \%$, but the roll-off angle is about $14^{\circ}$, slightly above the acceptable range for self-cleaning behavior (below $10^{\circ}$ ). At CNF concentrations above $30 \mathrm{wt} . \%$, the water roll-off angles are well within the self-cleaning regime. Oil droplet mobility is observed only at $60 \mathrm{wt} . \% \mathrm{CNF}$ loading, with the roll-off angle $\sim 9^{\circ}$ indicating self-cleaning behavior. In lower CNF loadings, the coatings display a "sticky" oleophobic surface (no droplet rolling) even when static CAs reach almost up to the superoleophobic threshold (for example at 40 wt.\%). These films also display the additional functionality of electrical conductivity (Fig. 5b); this quantity increases from $63 \mathrm{~S} / \mathrm{m}$ (at 10 wt. \% CNF) to $940 \mathrm{~S} / \mathrm{m}$ (at 60 wt. \% CNF) being well within the electromagnetic interference (EMI) shielding range [5].

In order to study the effect of underlying substrate roughness on coating repellency, thin conformal coatings were deposited on silicon carbide sand papers of different grit sizes. Sand papers of grit size 280, 600, 1200 and 1500 were used for this purpose with corresponding mean surface roughness of $45,15,9$, and $5 \mu \mathrm{m}$ respectively. Figures $6 \mathrm{a}$, b display the morphology of a 25 wt. \% CNF-containing polymer composite coating deposited by spray on a 600 grit sand paper substrate. Highly microporous polymer films with pores separated by thin polymer walls (sponge-like) are known as polymer foams; the associated surface morphology is commonly referred to as microcellular morphology [35]. Formation of such microcellular morphology is seen in Figs 6a, b and is attributed to the underlying substrate roughness, as compared to the morphology on a smooth glass substrate (Fig. 4a) which is lacking the micro-porous feature. Figure 6c displays the same 600 grit sand paper substrate in its bare state. In order to allow SEM 
observation, the sand paper sample was first coated with a 5-nm thick layer of platinum/palladium. Comparison of Figs $6 \mathrm{~b}$ and $6 \mathrm{c}$ reveals the conformal character of the deposited CNF/polymer film.
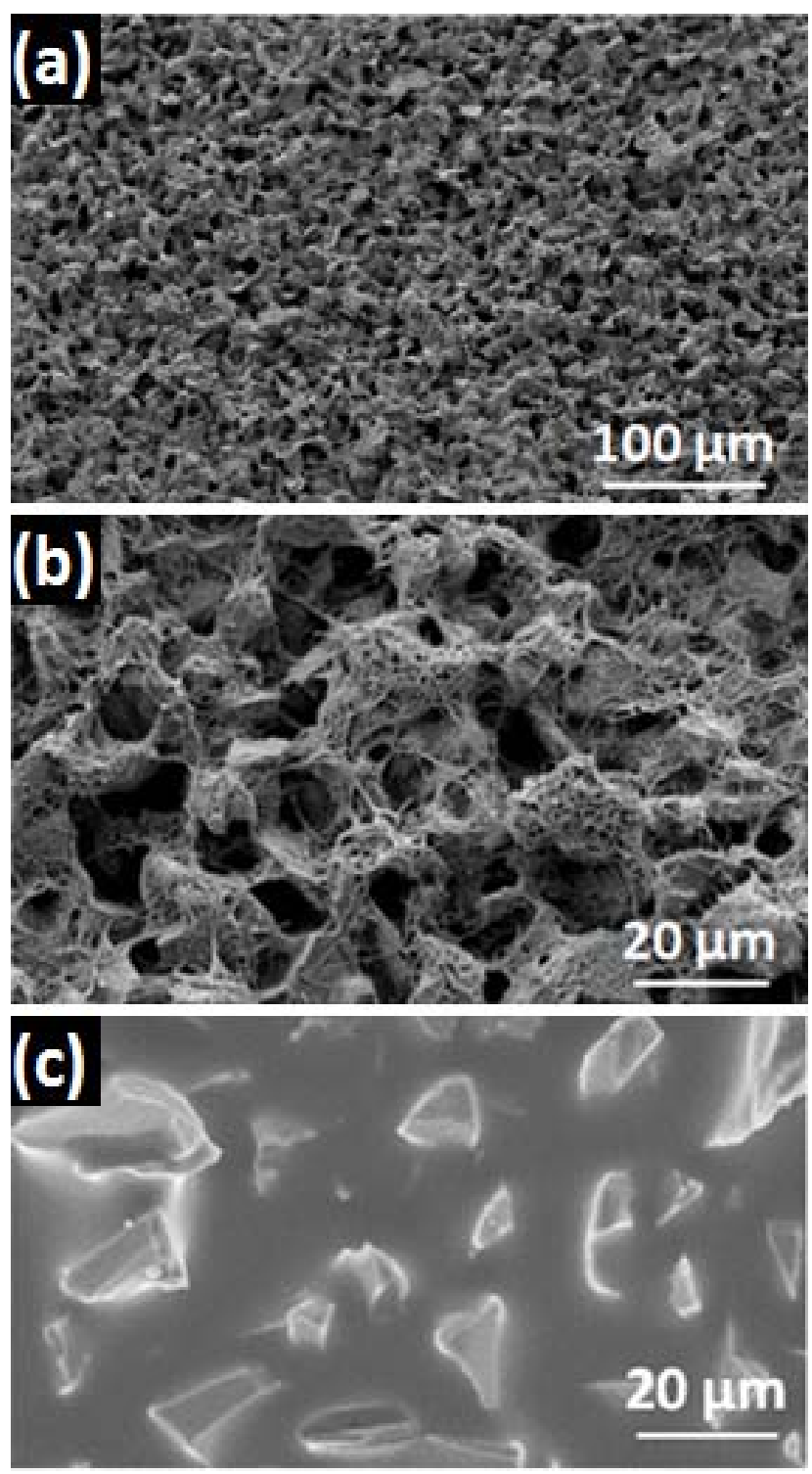

Fig. 6 - (a) Scanning electron microscope image showing morphology of a conformal superhydrophobic CNF/PMC composite (25 wt. \% CNF in total solids) spray-deposited on a 600 grit sand paper (average roughness $15 \mu \mathrm{m}$ ). (b) Higher magnification image showing coverage of sand paper micro roughness with the CNF/PMC composite web. (c) SEM image showing surface morphology of the bare 600 grit sand paper. 
(a)

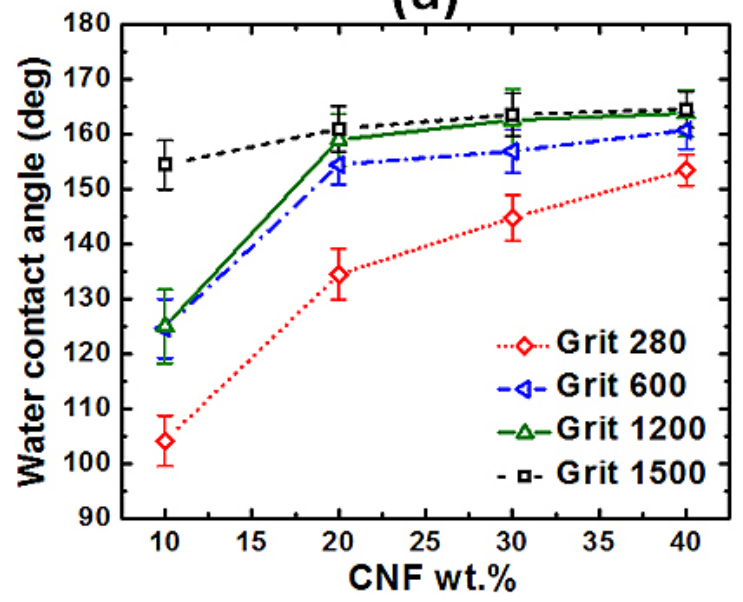

(b)

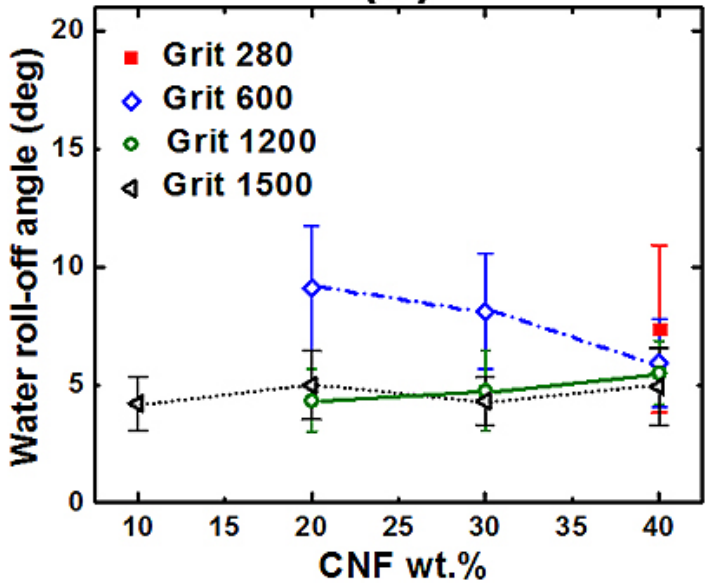

Fig. 7 - Variation of water (a) sessile contact angle and (b) droplet roll-off angle with CNF loading for conformal composite coatings deposited on sand papers of different micro-roughness (grit size in legend). Increasing grit size indicates reduced roughness.

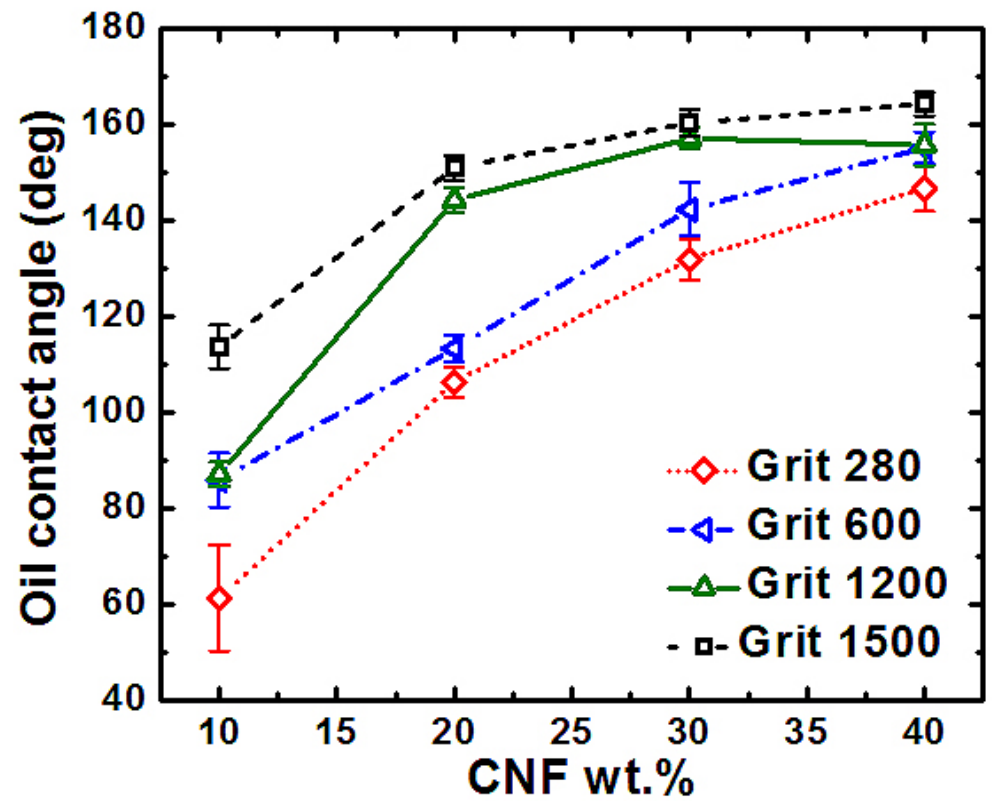

Fig. 8 - Variation of oil static contact angle with CNF loading for conformal composite coatings deposited on sand papers of varying micro-roughness (grit size in legend).

Detailed water sessile contact angles and droplet roll-off angles as a function of CNF concentration in total solids and substrate roughness are presented in Fig. 7. For any given 
composition, water CA increases with decreasing mean substrate roughness, while water droplet mobility increases (i.e. roll-off angle decreases) with decreasing roughness. In particular, significant changes are observed when the mean substrate roughness reduces from $45 \mu \mathrm{m}$ to 15 $\mu \mathrm{m}$ for all composite films. Only for the finest sand paper (5 $\mu \mathrm{m}$ mean surface roughness), superhydrophobic self-cleaning behavior is observed at all CNF loadings. For the roughest substrate with surface roughness of $45 \mu \mathrm{m}$, superhydrophobic self-cleaning behavior is attained only at the highest CNF loading (40 wt.\%). For the other two substrates with mean surface roughness between 9 and $15 \mu \mathrm{m}$, self-cleaning superhydrophobic behavior is observed for CNF concentration at or above 20 wt. \%, as compared to the 30 wt. \% threshold required for such behavior on the films applied on smooth glass substrates (Fig. 5). This observation confirms the importance of underlying substrate texture on superhydrophobic behavior.

In the case of oil repellency (Fig. 8), for substrate roughness below $15 \mu \mathrm{m}$, significant jumps in static CAs are observed as the CNF concentration rises from $10 \mathrm{wt}$ \% to $20 \mathrm{wt}$. \%. On sand papers with grit sizes of 1200 (9 $\mu \mathrm{m}$ mean roughness) or $1500(5 \mu \mathrm{m})$, composites with CNF concentrations near or above 20 wt. \% resulted in superoleophobic behavior with static oil CAs exceeding $150^{\circ}$. However, oil mobility on these apparently superoleophobic films was not observed (no droplet rolling even at $90^{\circ}$ tilt angles), thus classifying these films as "sticky" superoleophobic composites. We argue that this behavior is due to the disappearance of microcellular structure when the mean substrate roughness decreases. Based on an earlier study of PMC/zinc oxide composites [3], self-cleaning superoleophobic behavior was observed when the PMC polymer matrix assumed microcellular structure, as created by a controlled solvent inversion technique during spray atomization [3]. Inspection of the ESEM micrographs in Fig. 9 
indeed shows that the microcellular morphology is absent on coatings applied on the 1200 and 1500 grit (finest) substrates. As mentioned earlier, self-cleaning, low-surface-tension liquid (oil) repellency can only be achieved when dual-scale (micro/nano), uniformly-distributed roughness features with strong surface curvatures are created on very low surface energy (perfluorinated) surfaces. Absence of such features on the 1200 and 1500 grit surfaces in Fig. 9 probably causes increases in oil CA hysteresis, although the existing surface texture -due to randomly assembled CNFs in the PMC polymer matrix- appears to be sufficient to cause high static oil CAs. Nonetheless, even though the corresponding static oil contact angles are high, oil droplets tend to stick on these coated surfaces [36].
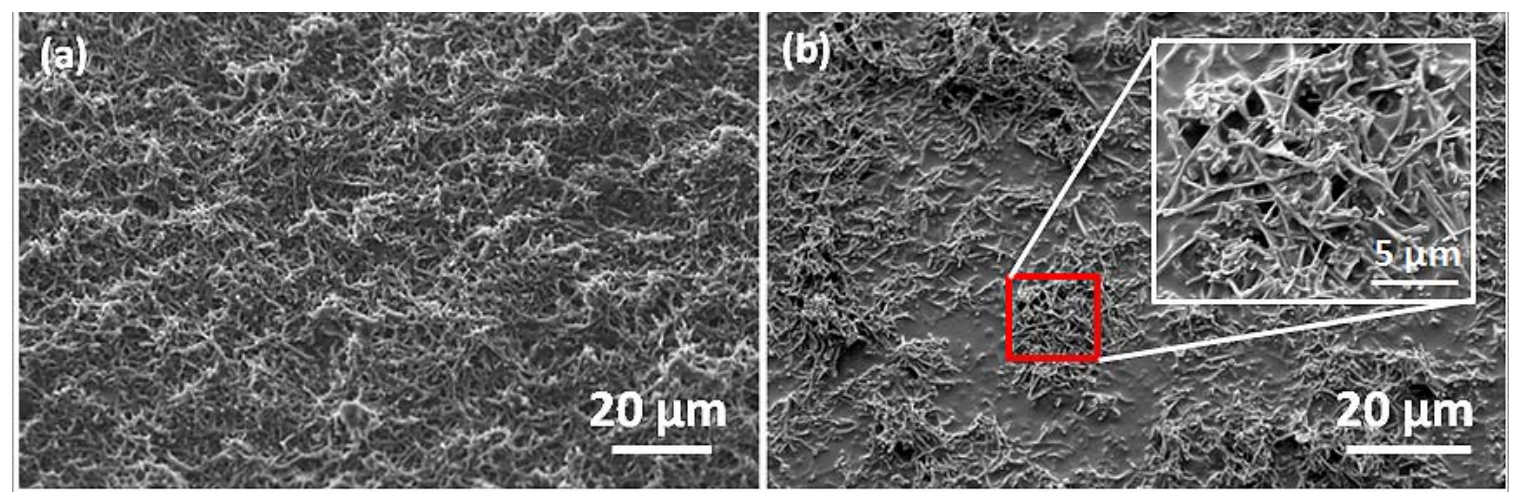

Fig. 9 - Scanning electron micrographs showing surface morphology of CNF/PMC (25 wt. \% CNF in solids) CNF/PMC composite films spray cast and dried on (a) 1200 grit, and (b) 1500 grit sand paper surfaces. The inset in (b) shows an enlargement of the marked area.

\subsection{Microscale Application: Ink-Jet Printed Patterns}

Inkjet printing is an attractive method for depositing spatially alternating wettable and nonwettable patterns [37]. The dispersions developed for the CNF/PMC composite fabrication cannot be used for ink-jet printing purposes, as the long CNFs cause frequent device orifice clogging. Instead, we produced carbon nanowhisker-PMC ink suspensions and generated 
conducting and liquid repellent line patterns with feature size of $100 \mu \mathrm{m}$. Figure 10 shows ink-jet printed superhydrophobic micro-ribbons generated on a Mylar substrate from a dilute carbon nanowhisker/PMC (wt. ratio of 1:1) dispersion. The printing was performed in the drop-ondemand mode at a dispensing frequency of $500 \mathrm{~Hz}$. The device orifice diameter was $60 \mu \mathrm{m}$, generating monodisperse droplets with diameters in the range $70-75 \mu \mathrm{m}$. The spot pitch was maintained constant at $40 \mu \mathrm{m}$, translating into a printing speed of $20 \mathrm{~mm} / \mathrm{s}$. The distance of the device orifice from the substrate was $0.5 \mathrm{~mm}$. Although the dark streaks in Fig. 10 show spatial non-uniformities caused during drying (coffee stain effect [38]), the lines were confirmed to be conductive. The intermittent area in between the dark lines in Fig. 10 has the wetting properties of the underlying substrate, thus requiring no further treatment. The example of Fig. 10 shows the potential of the present technique, but also displays the spatial uniformity challenges that must be overcome when applying these linear patterns $[39,40]$. Such patterns may be of value in microelectronic applications where conductive lines need also be liquid repellent. These composite lines are both electrically and thermally conductive due to presence of CNFs as filler. Patterning these composite films in specific geometric shapes, different non-metallic electromagnetic devices (such as polarizers, band-pass filters, etc.) can be prepared; due to their high liquid repellency, these devices will be corrosion resistant, offering an advantage over metals. 


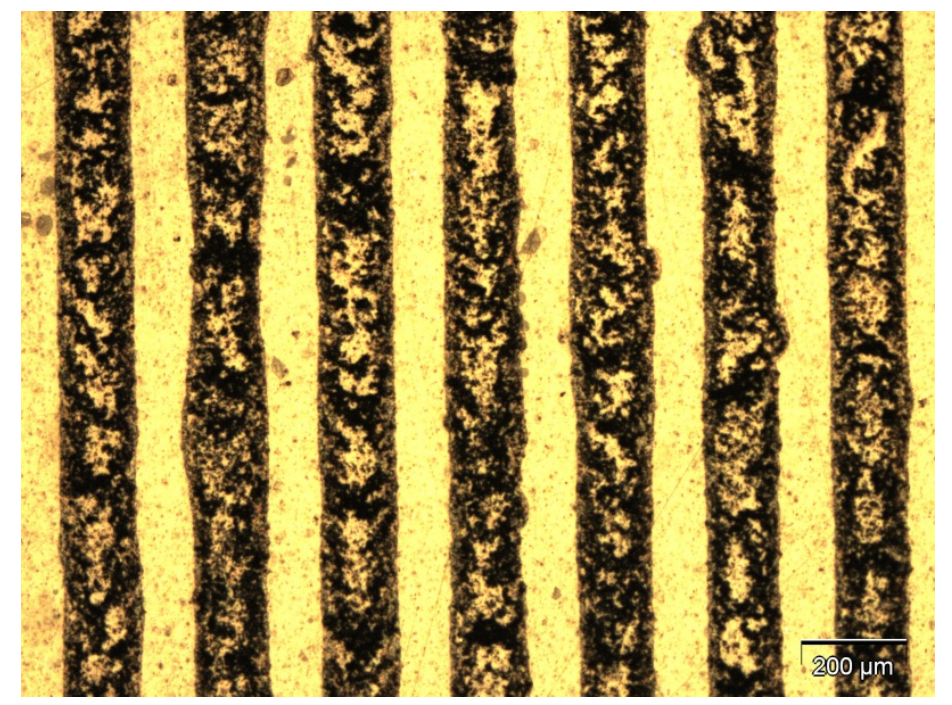

Fig. 10 - Hydrophobic and conducting carbon/fluoropolymer composite lines printed on a hydrophilic Mylar substrate (yellow background) from a dilute carbon nanowhisker/PMC solution using an ink-jet nozzle of 60 $\mu \mathrm{m}$ dia. The carbon nanowhisker concentration in the dried composite film is 50 wt. \%. The dark areas depict high content of nanowhiskers, while the bright areas indicate lack of the particle filler.

\section{Conclusion}

We developed a simple CNF/polymer dispersion formulation that can be applied over large areas by spray to produce electrically conducting, composite films of tunable oil and water wettability, reaching well into the super-repellent regime. Long CNFs (>100 $\mu \mathrm{m}$ pre-sonication) were effectively dispersed in a multi-component solvent system comprising formic acid, acetone and water with the addition of a commercially available aqueous fluoropolymer (PMC) dispersion. The effects of CNF and polymer concentration on the degree of water and oil repellency were evaluated by means of static contact angle measurements. Liquid mobility was assessed in terms of droplet roll-off angles on inclined surfaces. Conformal composite films were spray cast on substrates with varying micro roughness (sand papers with 280-1500 grit roughness) to examine 
qualitatively the effect of underlying substrate microtexture on coating morphology, as well as on water/oil repellency and droplet mobility. The present CNF/PMC composite films can find application as EMI shielding materials, for prevention of surface ice accumulation (via the local addition of heat), or as water-oil separation materials. A microscale application was also demonstrated by ink-jetting hydrophobic composite linear micropatterns using shorter-length carbon fibers (nanowhiskers), which allow long-term ink-jet operation without device clogging. Such fine patterns have potential in microelectronic applications.

\section{Acknowledgement}

We thank DuPont Inc. for providing the Capstone ${ }^{\circledR}$ ST-100 product and Applied Sciences Inc, for providing CNF samples of Pyrograf III; PR-24-XT-HHT. The authors acknowledge MicroFab Technologies for providing equipment and personnel for the ink-jetting experiments. 


\section{References}

1. Tiwari MK, Bayer IS, Jursich GM, Schutzius TM, Megaridis CM. Highly liquid-repellent, large-area, nanostructured poly(vinylidene fluoride)/poly(ethyl 2-cyanoacrylate) composite coatings: Particle filler effects. ACS Applied Materials \& Interfaces 2010;2:1114-9.

2. Manoudis PN, Karapanagiotis I, Tsakalof A, Zuburtikudis I, Panayiotou C. Superhydrophobic composite films produced on various substrates. Langmuir 2008; 24:11225-32.

3. Steele A, Bayer IS, Loth E. Inherently superoleophobic nanocomposite coatings by spray atomization. Nano Letters 2009;9:501-5.

4. Bayer IS, Tiwari MK, Megaridis CM. Biocompatible poly(vinylidene fluoride)/cyanoacrylate composite coatings with tunable hydrophobicity and bonding strength. Applied Physics Letters 2008;93:173902.

5. Das A, Hayvaci HT, Tiwari MK, Bayer IS, Erricolo D, Megaridis CM. Superhydrophobic and conductive carbon nanofiber/PTFE composite coatings for EMI shielding. Journal of Colloid and Interface Science 2011;353:311-5.

6. Bayer IS, Steele A, Martorana PJ, Loth E. Fabrication of superhydrophobic polyurethane/organoclay nano-structured composites from cyclomethicone-in-water emulsions. Applied Surface Science 2010;257:823-6. 
7. Bayer IS, Steele A, Martorana PJ, Loth E, Miller L. Superhydrophobic cellulose-based bio nanocomposite films from Pickering emulsions. Applied Physics Letters 2009;94: 163902.

8. Bayer I.S, Steele A, Martorana P, Loth E, Robinson SJ, Stevenson D. Biolubricant induced phase inversion and superhydrophobicity in rubber-toughened biopolymer/organoclay nanocomposites. Applied Physics Letters 2009;95:063702.

9. Bayer IS, Brown A, Steele A, Loth E. Transforming anaerobic adhesives into highly durable and abrasion resistant superhydrophobic organoclay nanocomposite films: A new hybrid spray adhesive for tough superhydrophobicity. Applied Physics Express 2009;2:125003.

10. Zhao J, Piao G, Wang X, Lian J, Wang Z, Hu H et al. Improved dispersion of PEGfunctionalized carbon nanofibers in toluene. Materials Science and Engineering C 2009; 29:7425.

11. Chen H, Muthuraman H, Stokes P, Zou JH, Liu X, Wang JH et al. Dispersion of carbon nanotubes and polymer nanocomposite fabrication using trifluoroacetic acid as a co-solvent. Nanotechnology 2007;18:415606.

12. Tibbetts GG, Lake ML, Strong KL, Rice BP. A review of the fabrication and properties of vapor-grown carbon nanofiber/ polymer composites. Composites Science and Technology 2007;67:1709-18. 
13. Wang K, Hu NX, Xu G, Qi Y. Stable superhydrophobic composite coatings made from an aqueous dispersion of carbon nanotubes and a fluoropolymer. Carbon 2011;49: 1769-74.

14. Gao LC, McCarthy TJ. The "lotus effect" explained: Two reasons why two length scales of topography are important. Langmuir 2006;22:2966-67.

15. Beecher P, Servati P, Rozhin A, Colli A, Scardaci V, Pisana S et al. Ink-jet printing of carbon nanotube thin film transistors. Journal of Applied Physics 2007;102:043710.

16. Dragoman M, Flahaut E, Dragoman D, Al Ahmad M, Plana R. Writing simple RF electronic devices on paper with carbon nanotube ink. Nanotechnology 2009;20:375203.

17. Elwi TA, Al-Rizzo HM, Rucker DG, Dervishi E, Li ZR, Biris AS. Multi-walled carbon nanotube-based RF antennas. Nanotechnology 2010;21:045301.

18. Han X, Janzen DC, Vaillancourt J, Lu X. Printable high-speed thin-film transistor on flexible substrate using carbon nanotube solution. Micro \& Nano Letters 2007;2:96-8.

19. Hsieh GW, Li FM, Beecher P, Nathan A, Wu YL, Ong BS et al. High performance nanocomposite thin film transistors with bilayer carbon nanotube-polythiophene active channel by ink-jet printing. Journal of Applied Physics 2009;106:123706. 
20. Jones CS, Lu XJ, Renn M, Stroder M, Shih WS. Aerosol-jet-printed, high-speed, flexible thin-film transistor made using single-walled carbon nanotube solution. Microelectronic Engineering 2010;87:434-7.

21. Kordas K, Mustonen T, Toth G, Jantunen H, Lajunen M, Soldano C et al. Inkjet printing of electrically conductive patterns of carbon nanotubes. Small 2006;2:1021-5.

22. Okimoto H, Takenobu T, Yanagi K, Shimotani H, Miyata Y, Kataura H et al. Low-voltage operation of ink-jet-printed single-walled carbon nanotube thin film transistors. Japanese Journal of Applied Physics 2010;49:02BD09.

23. Takenobu T, Miura N, Lu SY, Okimoto H, Asano T, Shiraishi M et al. Ink-jet printing of carbon nanotube thin-film transistors on flexible plastic substrates. Applied Physics Express 2009;2:025005.

24. Vaillancourt J, Zhang HY, Vasinajindakaw P, Xia HT, Lu XJ, Han XL et al. All ink-jetprinted carbon nanotube thin-film transistor on a polyimide substrate with an ultrahigh operating frequency of over 5 GHz. Applied Physics Letters 2008;93:243301.

25. Wei T, Ruan J, Fan Z, Luo G, Wei F. Preparation of a carbon nanotube film by ink-jet printing. Carbon 2007;45:2712-2716. 
26. Ozkan T, Naraghi M, Chasiotis I. Mechanical properties of vapor grown carbon nanofibers. Carbon 2010;48:239-244.

27. Pierce E, Carmona F.J, Amirfazli A. Understanding of sliding and contact angle results in tilted plate experiments. Colloids Surfaces A 2008;323:73-82.

28. Tuteja A, Choi W, Ma M, Mabry JM, Mazzella SA, Rutledge GC. Designing superoleophobic surfaces. Science 2007;318:1618-1622.

29. Wang S, Zhang Y, Abidi N, Cabrales L. Wettability and surface free energy of graphene films. Langmuir 2009;25:11078-11081.

30. Lin JJ, Chu CC, Chiang ML, Tsai WC. Manipulating assemblies of high-aspect-ratio clays and fatty amine salts to form surfaces exhibiting a lotus effect. Adv. Mater. 2006;18:3248-3252.

31. Ming Z, Jian L, Chunxia W, Xiaokang Z, Lan C. Fluid drag reduction on superhydrophobic surfaces coated with carbon nanotube forests (CNTs). Soft Matter 2011;7:4391-4396.

32. Sethi S, Dhinojwala A. Superhydrophobic conductive carbon nanotube coatings for steel. Langmuir 2009;25:4311-4313.

33. Morita M, Ogisu H, Kubo M. Surface properties of perfluoroalkylethyl acrylate/n-alkyl acrylate copolymers. Journal of Applied Polymer Science 1999;73:1741-1749. 
34. Israelachvili JN. Intermolecular and surface forces. 2nd ed. London: Academic Press; 1991: $32,125,132$.

35. Zhang HB, Yan Q, Zheng WG, He Z, Yu ZZ. Tough graphene-polymer microcellular foams for electromagnetic interference shielding. ACS Applied Materials and Interfaces 2011;3:918924.

36. Extrand CW, Gent AN. Retention of liquid drops by solid surfaces. Journal of Colloid and Interface Science 1990;138:431-442.

37. Brinkmann M, Lipowsky R. Wetting morphologies on substrates with striped surface domains. Journal of Applied Physics 2002;92:4296-4306.

38. Deegan RD, Bakajin O, Dupont TF, Huber G, Nagel SR, Witten TA. Capillary flow as the cause of ring stains from dried liquid drops. Nature 1997;389:827-829.

39. Szczech JB, Megaridis CM, Gamota DR, Zhang J. Fine-Line conductor manufacturing using advanced drop-on-demand PZT printing technology. IEEE Transactions on Electronics Packaging Manufacturing 2002;25:26-33. 
This article appeared in Carbon, Vol. 50, pp. 1346-1354 (2012)

40. Szczech JB, Megaridis CM, Zhang J, Gamota DR. Ink jet processing of metallic nanoparticle suspensions for electronic circuitry fabrication. Microscale Thermophysical Engineering 2004;8:327-339. 